\title{
Strong Pullback Attractors for Nonautonomous Suspension Bridge Equations
}

\author{
Wenchao Ju and Xuan Wang \\ College of Mathematics and Statistics, Northwest Normal University, Lanzhou, Gansu 730070, China \\ Correspondence should be addressed to Wenchao Ju; juwenchaojwc@126.com
}

Received 1 December 2013; Accepted 8 January 2014; Published 9 March 2014

Academic Editors: F. Ding and C.-H. Lien

Copyright (C) 2014 W. Ju and X. Wang. This is an open access article distributed under the Creative Commons Attribution License, which permits unrestricted use, distribution, and reproduction in any medium, provided the original work is properly cited.

We prove the existence of a pullback $\mathscr{D}$-attractor in $D(A) \times V$ for the nonautonomous suspension bridge equations.

\section{Introduction}

In this paper, we consider the following nonautonomous suspension bridge equation:

$$
\begin{gathered}
u_{t t}+\Delta u+\mu u_{t}+k u^{+}+g(u)=f(x, t), \quad \text { in } \Omega \times \mathbb{R}_{\tau}, \\
u(x, t)=\Delta u(x, t)=0, \quad \text { on } \partial \Omega \times \mathbb{R}_{\tau}, \\
u(x, \tau)=u_{1}(x), \quad u_{t}(x, \tau)=u_{2}(x), \quad x \in \Omega,
\end{gathered}
$$

where $\Omega$ is a bounded domain of $\mathbb{R}^{2}$ with a smooth boundary $\partial \Omega, u(x, t)$ is an unknown function, which could represent the deflection of the road bed in the vertical plane, $\mathrm{ku}^{+}$ represents the restoring force, $k$ denotes the spring constant, $\mu u_{t}$ represents the viscous damping, and $\mu$ is a given positive constant.

Suspension bridge equations have been posed as a new problem in the field of nonlinear analysis [1] by Lazer and Mckenna in 1990. There are many results for the problem (1) (cf. [1-8]), for instance, the existence, multiplicity, and properties of the travelling wave solutions, and so forth. About the long-time behavior of suspension bridge equations, for the autonomous case, in $[9,10]$, the authors have discussed long-time behavior of the solutions of the problem on $\mathbb{R}^{2}$ and obtained the existence of global attractors in the space $H_{0}^{2}(\Omega) \times L^{2}(\Omega)$ and $D(A) \times H_{0}^{2}(\Omega)$.

Caraballo et al. advanced the concept of the pullback $\mathscr{D}$ attractor in [11], and the existence of the pullback attractors was proved under the assumptions of asymptotic compactness and existence of a family of absorbing sets. Recently, Park and Kang [12] studied the pullback $\mathscr{D}$-attractor for suspension bridge equations in the weak space $H_{0}^{2}(\Omega) \times L^{2}(\Omega)$. Motivated by the ideas of $[11,13]$, we study the existence of a strong pullback $\mathscr{D}$-attractor for the nonautonomous suspension bridge equations in the strong topological space $D(A) \times H_{0}^{2}(\Omega)$.

The nonlinear functions $g \in C^{2}(\mathbb{R}, \mathbb{R})$ satisfy the following assumptions:

$$
\begin{gathered}
\liminf _{|u| \rightarrow \infty} \frac{G(u)}{u^{2}} \geqslant 0, \quad G(u)=\int_{0}^{u} g(\zeta) d \zeta, \\
|g(s)| \leqslant C\left(1+|s|^{p}\right), \quad \forall p \geqslant 1, \\
\liminf _{|u| \rightarrow \infty} \frac{u g(u)-C_{0} G(u)}{u^{2}} \geqslant 0,
\end{gathered}
$$

where constant $C, C_{0}>0$.

With the usual notation, we introduce the spaces $H=$ $L^{2}(\Omega), V=H_{0}^{2}(\Omega), D(A)=u \in\left\{H_{0}^{2}(\Omega) \mid A u \in L^{2}(\Omega)\right\}$, where $A=\Delta^{2}$. We equip these spaces with inner product and norm $\langle\cdot, \cdot\rangle,|\cdot|,\langle\cdot, \cdot\rangle_{1},\|\cdot\|_{1}$, and $\langle\cdot, \cdot\rangle_{2},\|\cdot\|_{2}$, respectively:

$$
\begin{gathered}
\langle u, v\rangle=\int_{\Omega} u(x) v(x) d x \\
|u|^{2}=\int_{\Omega}|u(x)|^{2} d x, \quad \forall u, v \in H ;
\end{gathered}
$$




$$
\begin{gathered}
\langle u, v\rangle_{1}=\int_{\Omega} \Delta u(x) \Delta v(x) d x \\
\|u\|_{1}^{2}=\int_{\Omega}|\Delta u(x)|^{2} d x, \quad \forall u, v \in V \\
\langle u, v\rangle_{2}=\int_{\Omega} \Delta^{2} u(x) \Delta^{2} v(x) d x \\
\|u\|_{2}^{2}=\int_{\Omega}\left|\Delta^{2} u(x)\right|^{2} d x, \quad \forall u, v \in D(A) .
\end{gathered}
$$

Obviously, we have

$$
D(A) \subset V \subset H=H^{*} \subset V^{*}
$$

where $H^{*}, V^{*}$ is dual space of $H, V$, respectively; the injections are continuous and each space is dense in the following one. have

Choosing $\lambda=\min \left\{\lambda_{1}, \lambda_{1}^{2}\right\}$, by the Poincaré inequality, we

$$
\begin{gathered}
\|z\|_{\mathscr{C}_{0}}=\left\|\left(u, u_{t}\right)\right\|_{\mathscr{E}_{0}}=\left\{\|u\|_{1}^{2}+\left|u_{t}\right|^{2}\right\}^{1 / 2}, \\
\|z\|_{\mathscr{E}_{1}}=\left\|\left(u, u_{t}\right)\right\|_{\mathscr{E}_{1}}=\left\{\|u\|_{2}^{2}+\left\|u_{t}\right\|_{1}^{2}\right\}^{1 / 2} .
\end{gathered}
$$

We introduce the Hilbert spaces

$$
\mathscr{E}_{0}=V \times H, \quad \mathscr{E}_{1}=D(A) \times V
$$

and endow this space with norm

$$
\begin{gathered}
\|z\|_{\mathscr{E}_{0}}=\left\|\left(u, u_{t}\right)\right\|_{\mathscr{E}_{0}}=\left\{\|u\|_{1}^{2}+\left\|u_{t}\right\|^{2}\right\}^{1 / 2}, \\
\|z\|_{\mathscr{C}_{1}}=\left\|\left(u, u_{t}\right)\right\|_{\mathscr{E}_{1}}=\left\{\|u\|_{2}^{2}+\left\|u_{t}\right\|_{1}^{2}\right\}^{1 / 2} .
\end{gathered}
$$

This paper is organized as follows. At first, in Section 2, we recall some preliminaries and results concerning the pullback attractor. Then, in Section 3, we prove our main result about the existence of pullback $\mathscr{D}$-attractor for the nonautonomous dynamical system generated by the solution of (1).

\section{Notation and Preliminaries}

Let $(E, d)$ be a complete metric space, $(Q, \rho)$ be a metric space which will be called the parameter space. We define a nonautonomous dynamical system by a cocycle mapping $\phi: \mathbb{R}_{+} \times Q \times E$ which is driven by an autonomous dynamical system $\theta$ acting on a parameter space $Q$. Specifically, $\theta=$ $\left\{\theta_{t}\right\}_{t \in \mathbb{R}}$ is a dynamical system on $Q$; that is, is a group of homeomorphisms under composition on $Q$ with the properties that

(i) $\theta_{0}(q)=q$ for all $q \in Q$;

(ii) $\theta_{t+\tau}(q)=\theta_{t}\left(\theta_{\tau}(q)\right)$ for all $q \in Q, t, \tau \in \mathbb{R}$;

(iii) the mapping $(t, q) \rightarrow \theta_{t}(q)$ is continuous.
Definition 1. A mapping $\phi$ is said to be a cocycle on $E$ with respect to group $\theta$, if

(i) $\phi(0, q, x)=x$ for all $(q, x) \in Q \times E$;

(ii) $\phi(t+s, q, x)=\phi\left(s, \theta_{t}(q), \phi(t, q, x)\right)$ for all $s, t \in \mathbb{R}_{+}$ and all $(q, x) \in Q \times E$.

Let $\mathscr{P}(E)$ denote the family of all nonempty subsets of $E$, let $\mathscr{B}(E)$ be the set of all bounded subsets of $E$, and let $\mathscr{K}$ be the class of all families $\widehat{D}=\left\{D_{q}\right\}_{q \in Q} \subset \mathscr{P}(E)$. We consider a nonempty subclass $\mathscr{D} \in \mathscr{K}$.

Definition 2 (see [11]). Let $(\theta, \phi)$ be a nonautonomous dynamical system on $Q \times E$. $(\theta, \phi)$ is said to be pullback $\mathscr{D}$-asymptotically compact if, for any $q \in Q$, any $\widehat{D} \in \mathscr{D}$, and any sequences $t_{n} \rightarrow+\infty, x_{n} \in D_{\theta_{-t_{n}(q)}}$, the sequence $\phi\left(t_{n}, \theta_{-t_{n}}(q), x_{n}\right)$ possesses a convergent subsequence.

Definition 3 (see [11]). A family $\widehat{B}=\left\{B_{q}\right\}_{q \in Q} \in \mathscr{K}$ is said to be pullback $\mathscr{D}$-absorbing if, for each $q \in Q$ and $\widehat{D} \in \mathscr{D}$, there exists $t_{0}(q, \widehat{D}) \geqslant 0$ such that

$$
\phi\left(t, \theta_{-t}(q), D_{\theta_{-t}(q)}\right) \subset B_{q} \quad \forall t \geqslant t_{0}(q, \widehat{D}) .
$$

Definition 4 (see [11]). A family $\widehat{C}=\left\{C_{q}\right\}_{q \in Q} \in \mathscr{K}$ is said to be pullback $\mathscr{D}$-attracting if

$$
\begin{array}{r}
\lim _{t \rightarrow \infty} \operatorname{dist}\left(\phi\left(t, \theta_{-t}(q), D_{\theta_{-t}(q)}\right), C_{q}\right)=0 \\
\forall q \in Q, \quad \widehat{D} \in \mathscr{D},
\end{array}
$$

where $\operatorname{dist}(X, Y)=\sup _{x \in X} \inf _{y \in Y} d(x, y)$ is the Hausdorff semidistance between $X$ and $Y$.

Definition 5 (see [11]). A family $\widehat{A}=\left\{A_{q}\right\}_{q \in Q} \in \mathscr{K}$ is called a global pullback $\mathscr{D}$-attractor if it satisfies:

(i) $A_{q}$ is compact for any $q \in Q$;

(ii) $\widehat{A}$ is pullback $\mathscr{D}$-attracting;

(iii) $\widehat{A}$ is invariant; that is, $\phi\left(t, q, A_{q}\right)=A_{\theta_{t}(q)}$ for all $(t, q) \epsilon$ $\mathbb{R}_{+} \times Q$.

Definition 6 (see [14]). Let $(\theta, \phi)$ be a nonautonomous dynamical system on $Q \times E$. $(\theta, \phi)$ is said to be satisfying pullback $\mathscr{D}$-Condition $(C)$ if, for any $q \in Q, \widehat{D} \in \mathscr{D}$, and any $\epsilon>0$, there exist a $t_{0}=t_{0}(q, \widehat{D}, \epsilon) \geqslant 0$ and a finite dimensional subspace $E^{1}$ of $E$ such that

(i) $P\left(\cup_{t \geqslant t_{0}} \phi\left(t, \theta_{-t}(q), D_{\theta_{-t}(q)}\right)\right)$ is bounded;

(ii) $\left\|(I-P)\left(\cup_{t \geqslant t_{0}} \phi\left(t, \theta_{-t}(q), D_{\theta_{-t}(q)}\right)\right)\right\|_{E} \leqslant \epsilon$, where $P$ : $E \rightarrow E^{1}$ is a bounded projector. 
Lemma 7 (see [14]). Let $(\theta, \phi)$ be a nonautonomous dynamical system on $Q \times E$. $(\theta, \phi)$ possesses a global pullback $\mathscr{D}$ attractor $\widehat{A}=\left\{A_{q}\right\}_{q \in Q}$ satisfying $A_{q}=\Lambda(\widehat{D}, q)$ if it

(i) has a pullback $\mathscr{D}$-absorbing set $\widehat{B}=\left\{B_{q}\right\}_{q \in Q} \in \mathscr{D}$;

(ii) satisfies pullback $\mathscr{D}$-Condition (C).

Theorem 8 (see [12]). Suppose that $k>0$ and the assumption (2)-(4) hold. $f(x, t) \in L_{\mathrm{loc}}^{2}(\mathbb{R}, H)$ satisfies (17). Then there exists a unique global pullback $\mathscr{D}_{\delta, \mathscr{E}_{0}}$-attractor in $\mathscr{E}_{0}$ for the nonautonomous dynamical system $(\theta, \phi)$ defined by (15). result.

We need the following lemmas in order to prove the main

Lemma 9 (see [14]). Let $H$ be an infinite dimensional Hilbert space and let the family $\left\{\omega_{i}\right\}_{i \in \mathbb{N}}$ be an orthonormal of $H$. Suppose $f(x, t) \in L_{\text {loc }}^{2}(\mathbb{R} ; H)$ and, for any $t \in \mathbb{R}$, $\int_{-\infty}^{t} e^{\sigma s}|f(x, s)|^{2} d s<\infty$ for some $\sigma \geqslant 0$. Then

$$
\lim _{n \rightarrow \infty} \int_{-\infty}^{t} e^{\sigma s}\left|\left(I-P_{n}\right) f(x, s)\right|_{H}^{2} d s=0, \quad \forall t \in \mathbb{R},
$$

where $P_{n}: H \rightarrow \operatorname{span}\left\{\omega_{1}, \omega_{2}, \ldots, \omega_{n}\right\}$ is the orthogonal projector.

Lemma 10 (see $[10])$. Suppose that $g \in C^{2}(\mathbb{R}, \mathbb{R})$ and satisfying (3). Then $g: D(A) \rightarrow V$ are continuous compact.

Lemma 11 (see [10]). Let $h\left(u, u_{t}\right)=g^{\prime}(u) u_{t}, g \in C^{2}(\mathbb{R}, \mathbb{R})$ satisfying (3), and $g(0)=0$. Then $h: D(A) \times V \rightarrow H$ is continuous compact.

\section{Pullback $\mathscr{D}$-Attractors for Nonautonomous Suspension Bridge Equations}

First, we give the following result.

Theorem 12. Suppose that $k>0$, satisfying (2)-(4), if $f \in$ $L_{\mathrm{loc}}^{2}\left(\mathbb{R}_{\tau} ; H\right)$ and $\left(u_{1}, u_{2}\right) \in \mathscr{E}_{0}$. Then system (1) has a unique solution:

$$
\left(u, u_{t}\right) \in C\left(\mathbb{R}_{\tau} ; \mathscr{E}_{0}\right),
$$

where $\mathbb{R}_{\tau}=[\tau, \infty)$. If, in addition, $f^{\prime} \in L^{2}\left(\tau, T ; L^{2}(\Omega)\right)$ and $\left(u_{1}, u_{2}\right) \in \mathscr{E}_{1}$, then

$$
\left(u, u_{t}\right) \in C\left(\mathbb{R}_{\tau} ; \mathscr{E}_{1}\right) .
$$

Moreover, the mapping $\left(u_{1}, u_{2}\right) \rightarrow\left(u(t), u_{t}(t)\right)$ is continuous in $\mathscr{E}_{1}$.

We can construct the nonautonomous dynamical system generated by problem (1) in $\mathscr{E}=\mathscr{E}_{0}\left(\right.$ or $\left.\mathscr{E}_{1}\right)$. We consider $Q=\mathbb{R}, \theta_{t}(\tau)=\tau+t$ and define

$$
\begin{aligned}
\phi\left(t, \tau, y_{0}\right)=y\left(t+\tau ; \tau, y_{0}\right) & =\left(u(t+\tau), u_{t}(t+\tau)\right), \\
\tau & \in \mathbb{R}, \quad t \geqslant 0, \quad y_{0} \in \mathscr{E} .
\end{aligned}
$$

The uniqueness of solution to problem (1) implies that

$$
\begin{array}{r}
\phi\left(t+s, \tau, y_{0}\right)=\phi\left(t, s+\tau, \phi\left(s, \tau, y_{0}\right)\right), \\
\tau \in \mathbb{R}, \quad s \geqslant 0, \quad y_{0} \in \mathscr{E} .
\end{array}
$$

Also, for all $\tau \in \mathbb{R}, t \geqslant 0$ the mapping $\phi(t, \tau, \cdot): \mathscr{E} \rightarrow \mathscr{E}$ defined by (15) is continuous. Consequently, the mapping $\phi$ defined by (15) is a continuous cocycle on $\mathscr{E}$.

Now, we assume that $f, f^{\prime} \in L_{\text {loc }}^{2}(\mathbb{R} ; H)$ and for any $t \in \mathbb{R}$,

$$
\int_{-\infty}^{t} e^{\delta s}|f(s)|^{2} d s<\infty
$$

where $0<\delta<\varrho$. Let $\Re_{\delta}$ be the set of all functions $r: \mathbb{R} \rightarrow$ $(0,+\infty)$ such that

$$
\lim _{t \rightarrow-\infty} e^{\delta t} r^{2}(t)=0
$$

and $\mathscr{D}_{\delta, \mathscr{E}_{0}}$ denotes the class of all families $\widehat{D}=\{D(t) ; t \in \mathbb{R}\} \subset$ $\mathscr{P}\left(\mathscr{E}_{0}\right)$ such that $D(t)=\bar{B}\left(0, r_{\widehat{D}}(t)\right)$ for some $r_{\widehat{D}} \in \mathfrak{R}_{\delta}$, where $\bar{B}\left(0, r_{\widehat{D}}(t)\right)$ is the closed ball in $\mathscr{E}_{0}$ centered at 0 with radius $r_{\widehat{D}}(t)$.

3.1. Pullback $\mathscr{D}$-Attractors in $\mathscr{E}_{1}$. In this subsection, we assume that $f, f^{\prime} \in L_{\text {loc }}^{2}(\mathbb{R} ; H)$ and for any $t \in \mathbb{R}$,

$$
\int_{-\infty}^{t} e^{\delta s}\left(|f(s)|^{2}+\left|f^{\prime}(s)\right|^{2}\right) d s<\infty
$$

Let $\mathscr{R}_{\delta}$ be the set of all functions $r: \mathbb{R} \rightarrow(0,+\infty)$, which satisfies (18) with $\delta \in(0, \varrho)$, and $\mathscr{D}_{\delta, \mathscr{C}_{1}}$ denotes the class of all families $\widehat{D}=\{D(t) ; t \in \mathbb{R}\} \subset \mathscr{P}\left(\mathscr{E}_{1}\right)$ such that $D(t) \subset$ $\bar{B}\left(0, r_{\widehat{D}}(t)\right)$ for some $r_{\widehat{D}} \in \mathscr{R}_{\delta}$, where $\bar{B}\left(0, r_{\widehat{D}}(t)\right)$ is the closed ball in $\mathscr{E}_{1}$ centered at 0 with $r_{\widehat{D}}(t)$.

Theorem 13. Suppose that $f, f^{\prime} \in L_{\text {loc }}^{2}(\mathbb{R} ; H)$ satisfy (19). Then, there exists a unique global pullback $\mathscr{D}_{\delta, \mathscr{E}_{1}}$-attractor in $\mathscr{E}_{1}$ for the nonautonomous dynamical system $(\theta, \phi)$ defined by (15).

Proof. By Lemma 7, we need to prove the existence of a pullback $\mathscr{D}_{\delta, \mathscr{E}_{1}}$-absorbing set belonging to $\mathscr{D}_{\delta, \mathscr{E}_{1}}$ and then show that the cocycle $\phi$ defined by (15) satisfies pullback $\mathscr{D}_{\delta, \mathscr{C}_{1}}$-Condition $(C)$.

Multiplying (1) by $A v(t)=A u_{t}(t)+\varrho A u(t)$ and integrating over $\Omega$, we have

$$
\begin{aligned}
\frac{1}{2} \frac{d}{d t} & \left(\|v\|_{1}^{2}+\|u\|_{2}^{2}\right)+\varrho\|u\|_{2}^{2}+(\mu-\varrho)\|v\|_{1}^{2} \\
& -\varrho(\mu-\varrho)\langle u, v\rangle_{1}+k\left\langle u^{+}, A v\right\rangle+\langle g(u), A v\rangle \\
& =\langle f(x, t), A v\rangle .
\end{aligned}
$$


Using the Hölder and Young inequalities, we obtain

$$
\begin{aligned}
\varrho\|u\|_{2}^{2} & +(\mu-\varrho)\|v\|_{1}^{2}-\varrho(\mu-\varrho)\langle u, v\rangle_{1} \\
& \geqslant \varrho\|u\|_{2}^{2}+(\mu-\varrho)\|v\|_{1}^{2}-\frac{\varrho(\mu-\varrho)}{\sqrt{\lambda}}\|u\|_{2} \cdot\|v\|_{1} \\
& \geqslant \varrho\|u\|_{2}^{2}+(\mu-\varrho)\|v\|_{1}^{2}-\frac{\varrho}{4}\|u\|_{2}^{2}-\frac{\varrho \mu^{2}}{\lambda}\|v\|_{1}^{2} \\
& \geqslant \frac{3 \varrho}{4}\|u\|_{2}^{2}+\left(\mu-\varrho-\frac{\varrho \mu^{2}}{\lambda}\right)\|v\|_{1}^{2} .
\end{aligned}
$$

We can easily see that

$$
\begin{aligned}
\left\langle k u^{+}, A v\right\rangle= & \left\langle k u^{+}, A u_{t}\right\rangle+\left\langle k u^{+}, \varrho A u\right\rangle \\
= & \frac{d}{d t} k\left\langle u^{+}, A u\right\rangle+k \varrho\left\langle u^{+}, A u\right\rangle-k\left\langle\left(u^{+}\right)_{t}, A u\right\rangle \\
& \geqslant \frac{d}{d t} k\left\langle u^{+}, A u\right\rangle+k \varrho\left\langle u^{+}, A u\right\rangle-\frac{\varrho}{16}\|u\|_{2}^{2} \\
& -\frac{4 k^{2}}{\varrho}\left|u_{t}\right|^{2} .
\end{aligned}
$$

According to (3), Theorem 8, and the Sobolev embedding theorem, we know that $g(u), g^{\prime}(u)$ are uniformly bounded in $L^{\infty}$. That is, there exists a constant $k_{0}>0$, such that

$$
|g(u)| \leqslant k_{0}, \quad\left|g^{\prime}(u)\right| \leqslant k_{0} .
$$

In view of the Hölder inequality and (23), we can know

$$
\begin{aligned}
\langle g(u), A v\rangle= & \left\langle g(u), A u_{t}\right\rangle+\langle g(u), \varrho A u\rangle \\
= & \frac{d}{d t}\langle g(u), A u\rangle-\left\langle g^{\prime}(u) u_{t}, A u\right\rangle \\
& +\varrho\langle g(u), A u\rangle \\
\geqslant & \frac{d}{d t}\langle g(u), A u\rangle+\varrho\langle g(u), A u\rangle \\
& -\int_{\Omega}\left|g^{\prime}(u)\right| \cdot\left|u_{t}\right| \cdot|A u| d x \\
\geqslant & \frac{d}{d t}\langle g(u), A u\rangle+\varrho\langle g(u), A u\rangle-\frac{\varrho}{16}\|u\|_{2}^{2} \\
& -\frac{4 k_{0}^{2}}{\varrho}\left|u_{t}\right|^{2}, \\
\langle f(x, t), A v\rangle= & \left\langle f(x, t), A u_{t}\right\rangle+\langle f(x, t), \varrho A u\rangle \\
= & \frac{d}{d t}\langle f(x, t), A u\rangle-\left\langle f^{\prime}(x, t), A u\right\rangle \\
& +\varrho\langle f(x, t), A u\rangle
\end{aligned}
$$

$$
\begin{aligned}
\leqslant & \frac{d}{d t}\langle f(x, t), A u\rangle+\varrho\langle f(x, t), A u\rangle \\
& +\frac{\varrho}{8}\|u\|_{2}^{2}+\frac{2}{\varrho}\left|f^{\prime}(x, t)\right|^{2} .
\end{aligned}
$$
we get

We choose $\varrho$ small enough, such that $\mu-\varrho-\left(\varrho \mu^{2} / \lambda\right) \geqslant \varrho / 2$;

$$
\begin{aligned}
& \frac{d}{d t}\left(\|v\|_{1}^{2}+\|u\|_{2}^{2}+2 k\left\langle u^{+}, A u\right\rangle\right.+2\langle g(u), A u\rangle \\
&-2\langle f(x, t), A u\rangle)+ \varrho\left(\|v\|_{1}^{2}+\|u\|_{2}^{2}\right. \\
&+2 k\left\langle u^{+}, A u\right\rangle \\
&+2\langle g(u), A u\rangle \\
&-2\langle f(x, t), A u\rangle) \\
& \leqslant\left(\frac{8 k^{2}}{\varrho}+\frac{8 k_{0}^{2}}{\varrho}\right)\left|u_{t}\right|^{2}+\frac{4}{\varrho}\left|f^{\prime}(x, t)\right|^{2} .
\end{aligned}
$$

On the other hand, by the Hölder and Young inequalities, (??) and (23), it follows that

$$
\begin{aligned}
& \frac{d}{d t}\left(\frac{1}{2}\|u\|_{2}^{2}+2 k\left\langle u^{+}, A u\right\rangle\right)=\frac{d}{d t}\left|\frac{1}{\sqrt{2}} A u+\sqrt{2} k u^{+}\right|^{2} \\
& -4 k^{2} \int_{\Omega}\left|u^{+}\right| \cdot\left|\left(u^{+}\right)_{t}\right| d x \\
& \geqslant \frac{d}{d t}\left|\frac{1}{\sqrt{2}} A u+\sqrt{2} k u^{+}\right|^{2} \\
& -\frac{2 k^{2}}{\lambda}\|u\|_{1}^{2}-2 k^{2}\left|u_{t}\right|^{2} \\
& \frac{d}{d t}\left(\frac{1}{2}\|u\|_{2}^{2}+2\langle g(u), A u\rangle-2\langle f(x, t), A u\rangle\right) \\
& \geqslant \frac{d}{d t}\left|\frac{1}{\sqrt{2}} A u+\sqrt{2} g(u)-\sqrt{2} f(x, t)\right|^{2} \\
& -4 \int_{\Omega}|g(u)| \cdot\left|g^{\prime}(u) u_{t}\right| d x \\
& -4 \int_{\Omega}|f(x, t)| \cdot\left|f^{\prime}(x, t)\right| d x \\
& +4 \int_{\Omega}\left|g^{\prime}(u) u_{t}\right| \cdot|f(x, t)| d x \\
& +4 \int_{\Omega}|g(u)| \cdot\left|f^{\prime}(x, t)\right| d x \\
& \geqslant \frac{d}{d t}\left|\frac{1}{\sqrt{2}} A u+\sqrt{2} g(u)-\sqrt{2} f(x, t)\right|^{2}-4 k_{0}^{2}-4 k_{0}^{2}\left|u_{t}\right|^{2} \\
& -4|f(x, t)|^{2}-4\left|f^{\prime}(x, t)\right|^{2},
\end{aligned}
$$




$$
\begin{aligned}
& \varrho\left(\frac{1}{2}\|u\|_{2}^{2}+2 k\left\langle u^{+}, A u\right\rangle\right) \\
& \geqslant \varrho\left|\frac{1}{\sqrt{2}} A u+\sqrt{2} k u^{+}\right|^{2}-2 \varrho k^{2}\left|u^{+}\right|^{2} \\
& \geqslant \varrho\left|\frac{1}{\sqrt{2}} A u+\sqrt{2} k u^{+}\right|^{2}-\frac{2 \varrho k^{2}}{\lambda}\|u\|_{1}^{2}, \\
& \varrho\left(\frac{1}{2}\|u\|_{2}^{2}+2\langle g(u), A u\rangle-2\langle f(x, t), A u\rangle\right) \\
& \geqslant \varrho\left|\frac{1}{\sqrt{2}} A u+\sqrt{2} g(u)-\sqrt{2} f(x, t)\right|^{2}-2 \varrho|g(u)|^{2} \\
& \quad-2 \varrho|f(x, t)|^{2}+4 \varrho \int_{\Omega}|g(u)| \cdot|f(x, t)| d x \\
& \geqslant \varrho\left|\frac{1}{\sqrt{2}} A u+\sqrt{2} g(u)-\sqrt{2} f(x, t)\right|^{2} \\
& \quad-4 \varrho k_{0}^{2}-4 \varrho|f(x, t)|^{2} .
\end{aligned}
$$

Therefore, combining (26)-(29), we get

$$
\begin{aligned}
& \frac{d}{d t}\left(\|v\|_{1}^{2}+\left|\frac{1}{\sqrt{2}} A u+\sqrt{2} k u^{+}\right|^{2}\right. \\
& \left.+\left|\frac{1}{\sqrt{2}} A u+\sqrt{2} g(u)-\sqrt{2} f(x, t)\right|^{2}\right) \\
& +\varrho\left(\|v\|_{1}^{2}+\left|\frac{1}{\sqrt{2}} A u+\sqrt{2} k u^{+}\right|^{2}\right. \\
& \left.\quad+\left|\frac{1}{\sqrt{2}} A u+\sqrt{2} g(u)-\sqrt{2} f(x, t)\right|^{2}\right) \\
& \leqslant\left(\frac{8 k^{2}}{\varrho}+\frac{8 k_{0}^{2}}{\varrho}+2 k^{2}+4 k_{0}^{2}\right)\left|u_{t}\right|^{2}+\frac{2 k^{2}}{\lambda}(1+\varrho)\|u\|_{1}^{2} \\
& \quad+4(1+\varrho)|f(x, t)|^{2}+4\left(\frac{1}{\varrho}+1\right)\left|f^{\prime}(x, t)\right|^{2} \\
& \quad+4(1+\varrho) k_{0}^{2} .
\end{aligned}
$$

Set

$$
\begin{gathered}
C_{1}=\max \left\{\frac{8 k^{2}}{\varrho}+\frac{8 k_{0}^{2}}{\varrho}+2 k^{2}+4 k_{0}^{2}, \frac{2 k^{2}}{\lambda}(1+\varrho)\right\}, \\
C_{2}=\max \left\{4(1+\varrho), 4\left(\frac{1}{\varrho}+1\right)\right\} .
\end{gathered}
$$

Thus, denote

$$
\begin{aligned}
y(t)= & \|v\|_{1}^{2}+\left|\frac{1}{\sqrt{2}} A u+\sqrt{2} k u^{+}\right|^{2} \\
& +\left|\frac{1}{\sqrt{2}} A u+\sqrt{2} g(u)-\sqrt{2} f(x, t)\right|^{2}, \quad \text { for } t \geqslant \tau .
\end{aligned}
$$

We have

$$
\begin{aligned}
\frac{d}{d t} y(t)+\varrho y(t) \leqslant & C_{1}\left(\|u\|_{1}^{2}+\left|u_{t}\right|^{2}\right) \\
& +C_{2}\left(|f(t)|^{2}+\left|f^{\prime}(t)\right|^{2}\right) \\
& +4(1+\varrho) k_{0}^{2} .
\end{aligned}
$$

By the Gronwall lemma, we have

$$
\begin{aligned}
y(t) \leqslant & e^{-\varrho \tau} y(t-\tau)+4(1+\varrho) k_{0}^{2} \int_{t-\tau}^{t} e^{-\varrho(t-s)} d s \\
& +C_{1} \int_{t-\tau}^{t} e^{-\varrho(t-s)}\left(\|u\|_{1}^{2}+\left|u_{t}\right|^{2}\right) d s \\
& +C_{2} \int_{t-\tau}^{t} e^{-\varrho(t-s)}\left(|f(t)|^{2}+\left|f^{\prime}(t)\right|^{2}\right) d s .
\end{aligned}
$$

Set

$$
C_{3}=\max \left\{1+2 \varrho^{2} \lambda^{-1}, 2\right\}, \quad \varrho_{0}=1+\left(\varrho^{2}+\frac{4 k^{2}}{\lambda}\right) \frac{1}{\lambda} .
$$

Then

$$
\begin{aligned}
\|u\|_{2}^{2}+\left\|u_{t}\right\|_{1}^{2} \leqslant & 4 C_{3} e^{-\varrho \tau}\left(\varrho_{0}\left\|u_{1}\right\|_{2}^{2}+\left\|u_{2}\right\|_{1}^{2}\right) \\
& +8 C_{3} e^{-\varrho \tau}\left(2\left|g\left(u_{1}\right)\right|^{2}+2|f(t-\tau)|^{2}\right) \\
& +2 C_{1} C_{3} \int_{t-\tau}^{t} e^{-\varrho(t-s)}\left(\|u\|_{1}^{2}+\left|u_{t}\right|^{2}\right) d s \\
& +2 C_{2} C_{3} \int_{t-\tau}^{t} e^{-\varrho(t-s)}\left(|f(t)|^{2}+\left|f^{\prime}(t)\right|^{2}\right) d s \\
& +8 C_{3}(1+\varrho) k_{0}^{2} \int_{t-\tau}^{t} e^{-\varrho(t-s)} d s \\
& +\frac{4 C_{3} k^{2}}{\lambda}\|u\|_{1}^{2}+8 C_{3}|g(u)|^{2}+8 C_{3}|f(t)|^{2} .
\end{aligned}
$$

From Theorem 8, we have

$$
\begin{aligned}
\|u\|_{1}^{2}+\left|u_{t}\right|^{2} \leqslant & c_{1} c_{2} e^{-\delta \tau}\left(\left\|u_{1}\right\|_{1}^{2}+\left|u_{2}\right|^{2}\right) \\
& +\frac{c_{1} e^{-\delta t}}{\alpha_{1}} \int_{s-\tau}^{\tau} e^{\delta \xi}|f(\xi)|^{2} d \xi+\frac{2 c_{1} \alpha M}{\delta}
\end{aligned}
$$

where

$$
\begin{gathered}
c_{1}=\max \left\{2,1+2 \alpha^{2} \lambda^{-1}\right\}, \\
c_{2}=\max \left\{2,1+\lambda^{-1}\left(2 \alpha^{2}+k\right)\right\},
\end{gathered}
$$


and then

$$
\begin{aligned}
& \int_{t-\tau}^{t} e^{-\varrho(t-s)}\left(\|u\|_{1}^{2}+\left|u_{t}\right|^{2}\right) d s \\
& \leqslant c_{1} c_{2} e^{-\delta \tau}\left(\left\|u_{1}\right\|_{1}^{2}+\left|u_{2}\right|^{2}\right) \int_{-\infty}^{t} e^{-\varrho(t-s)} d s+\frac{2 c_{1} \alpha M}{\delta \varrho} \\
& \quad+\frac{c_{1}}{\alpha_{1}}\left(\int_{-\infty}^{t} e^{\delta s}|f(s)|^{2} d s\right)\left(\int_{-\infty}^{t} e^{-\delta s} e^{-\varrho(t-s)} d s\right) \\
& \leqslant \frac{c_{1} c_{2}}{\varrho \lambda} e^{-\delta \tau}\left(\left\|u_{1}\right\|_{2}^{2}+\left\|u_{2}\right\|_{1}^{2}\right)+\frac{2 c_{1} \alpha M}{\delta \varrho}+\frac{c_{1}}{\alpha_{1}(\varrho-\delta)} \\
& \quad \times \int_{-\infty}^{t} e^{-\delta(t-s)}|f(s)|^{2} d s .
\end{aligned}
$$

Set

$$
\begin{gathered}
C_{4}=\max \left\{4 C_{3}, \frac{2 c_{1} c_{2} C_{1} C_{3}}{\varrho \lambda}\right\}, \\
C_{5}=\max \left\{2 C_{2} C_{3}, \frac{2 c_{1} C_{1} C_{3}}{\alpha_{1}(\varrho-\delta)}\right\}, \\
C_{6}=8 C_{3}\left(1+\frac{1}{\alpha}\right) k_{0}^{2}+\frac{4 c_{1} \alpha_{0} M C_{1} C_{3}}{\delta \alpha_{1}}+8 C_{3} k_{0}^{2} .
\end{gathered}
$$
have

Let $D=\{D(t)\}_{t \in \mathbb{R}} \in D_{\delta, \mathscr{C}_{1}}$. Combining (36) and (39), we

$$
\begin{gathered}
\left\|\phi\left(\tau, t-\tau, y_{0}\right)\right\|_{\mathscr{E}_{1}}^{2}=\|u\|_{2}^{2}+\left\|u_{t}\right\|_{1}^{2} \\
\leqslant C_{4}\left(e^{-\rho \tau}+e^{-\delta \tau}\right)\left(\left\|u_{1}\right\|_{2}^{2}+\left\|u_{2}\right\|_{1}^{2}\right) \\
+C_{5}\left(\int_{-\infty}^{t} e^{-\varrho(t-s)}\left(|f(s)|^{2}+\left|f^{\prime}(s)\right|^{2}\right) d s\right. \\
\left.\quad+\int_{-\infty}^{t} e^{-\delta(t-s)}|f(s)|^{2} d s\right) \\
+16 C_{3} e^{-\varrho \tau}|f(t-\tau)|^{2}+16 C_{3} k_{0}^{2} e^{-\varrho \tau} \\
+\frac{4 C_{3} k^{2}}{\lambda}\|u\|_{1}^{2}+8 C_{3}\|f(t)\|^{2}+C_{6},
\end{gathered}
$$

for all $y_{0} \in D(t-\tau), t \in \mathbb{R}$, and $\tau \geqslant 0$.

Set

$$
\begin{aligned}
\left(R_{\delta, \mathscr{E}_{1}}(t)\right)^{2}= & 2 C_{5}\left(\int_{-\infty}^{t} e^{-\varrho(t-s)}\left(|f(s)|^{2}+\left|f^{\prime}(s)\right|^{2}\right) d s\right. \\
& \left.+\int_{-\infty}^{t} e^{-\delta(t-s)}|f(s)|^{2} d s\right) \\
& +32 C_{3} k_{0}^{2} e^{-\varrho \tau}+\frac{8 C_{3} k^{2}}{\lambda}\left(R_{\delta, \mathscr{E}_{0}}(t)\right)^{2} \\
& +16 C_{3}\|f(t)\|^{2}+2 C_{6}
\end{aligned}
$$

and consider the family $\widehat{B}_{\delta, \mathscr{C}_{1}}$ of closed balls in $\mathscr{E}_{1}$ defined by

$$
B_{\delta, \mathscr{C}_{1}}(t)=\left\{z \in \mathscr{E}_{1} ;\|z\|_{\mathscr{E}_{1}} \leqslant R_{\delta, \mathscr{C}_{1}}(t)\right\}
$$

From (18) and (41), $\widehat{B}_{\delta, \mathscr{C}_{1}}$ is a pullback $\mathscr{D}_{\delta, \mathscr{E}_{1}}$-absorbing for the cocycle $\phi$ in $\mathscr{E}_{1}$.

Next, we show that the cocycle $\phi$ satisfies the pullback $\mathscr{D}_{\delta, \mathscr{E}_{1}}$-Condition $(C)$.

We assume that $\widetilde{\lambda}_{i}, i=1,2, \ldots$, are eigenvalue of operator $A$ in $D(A)$, satisfying

$$
0<\tilde{\lambda}_{1}<\tilde{\lambda}_{2} \leqslant \cdots \leqslant \tilde{\lambda}_{j} \leqslant \cdots, \quad \tilde{\lambda}_{j} \longrightarrow \infty \text {, as } j \longrightarrow \infty,
$$

$\widetilde{\omega}_{i}$ denotes eigenvector corresponding to eigenvalue $\widetilde{\lambda}_{i}, i=$ $1,2,3, \ldots$, which forms an orthogonal basis in $D(A)$, and at the same time they are also a group of canonical bases in $D(A)$ or $V$ and satisfy

$$
A \widetilde{\omega}_{i}=\widetilde{\lambda}_{i} \widetilde{\omega}_{i}, \quad \forall i \in \mathbb{N}
$$

Let $V_{m}=\operatorname{span}\left\{\widetilde{\omega}_{1}, \widetilde{\omega}_{2}, \ldots, \widetilde{\omega}_{m}\right\}$ and $P_{m}: V \rightarrow V_{m}$ is an orthogonal projector. For any $\left(u, u_{t}\right) \in \mathscr{E}_{1}$, we write

$$
\left(u, u_{t}\right)=\left(u_{1}, u_{1 t}\right)+\left(u_{2}, u_{2 t}\right) \text {, }
$$

where $\left(u_{1}, u_{1 t}\right)=\left(P_{m} u, P_{m} u_{t}\right)$.

Taking the scalar product with $A v_{2}(t)=A u_{2 t}(t)+\sigma A u_{2}(t)$ for (1) in $H$, we have

$$
\begin{aligned}
\frac{1}{2} \frac{d}{d t} & \left(\left\|v_{2}\right\|_{1}^{2}+\left\|u_{2}\right\|_{2}^{2}\right)+\sigma\left\|u_{2}\right\|_{2}^{2}+(\mu-\sigma)\left\|v_{2}\right\|_{1}^{2} \\
& -\sigma(\mu-\sigma)\left\langle u_{2}, v_{2}\right\rangle_{1}+k\left\langle u^{+}, A v_{2}\right\rangle+\left\langle g(u), A v_{2}\right\rangle \\
= & \left\langle f(x, t), A v_{2}\right\rangle .
\end{aligned}
$$

Similar to the estimate of (21) and (22), we have

$$
\begin{aligned}
\sigma & \left\|u_{2}\right\|_{2}^{2}+(\mu-\sigma)\left\|v_{2}\right\|_{1}^{2}-\sigma(\mu-\sigma)\left\langle u_{2}, v_{2}\right\rangle_{1} \\
& \geqslant \frac{3 \sigma}{4}\left\|u_{2}\right\|_{2}^{2}+\frac{\sigma}{2}\left\|v_{2}\right\|_{1}^{2}, \\
\left\langle k\left(u^{+}\right)_{2}, A v_{2}\right\rangle \geqslant & \frac{d}{d t} k\left\langle\left(u^{+}\right)_{2}, A u_{2}\right\rangle+k \sigma\left\langle\left(u^{+}\right)_{2}, A u_{2}\right\rangle \\
& -\frac{\varrho}{16}\left\|u_{2}\right\|_{2}^{2}-\frac{4 k^{2}}{\sigma}\left\|\left(u^{+}\right)_{2 t}\right\|^{2} .
\end{aligned}
$$


Moreover, we obtain

$$
\begin{aligned}
\left\langle g(u), A v_{2}\right\rangle= & \frac{d}{d t}\left\langle(g(u))_{2}, A u_{2}\right\rangle-\left\langle\left(g^{\prime}(u) u_{t}\right)_{2}, A u_{2}\right\rangle \\
& +\sigma\left\langle(g(u))_{2}, A u_{2}\right\rangle \\
\geqslant & \frac{d}{d t}\left\langle(g(u))_{2}, A u_{2}\right\rangle+\sigma\left\langle(g(u))_{2}, A u_{2}\right\rangle \\
& -\frac{\sigma}{16}\left\|u_{2}\right\|_{2}^{2}-\frac{4}{\sigma}\left|\left(g^{\prime}(u) u_{t}\right)_{2}\right|^{2}, \\
\langle(I- & \left.\left.P_{m}\right) f(x, t), A v_{2}\right\rangle \\
= & \frac{d}{d t}\left\langle f_{m}(x, t), A u_{2}\right\rangle-\left\langle f_{m}^{\prime}(x, t), A u\right\rangle \\
& +\sigma\left\langle f(x, t), A u_{2}\right\rangle \\
\leqslant & \frac{d}{d t}\left\langle f_{m}(x, t), A u_{2}\right\rangle+\sigma\left\langle f_{m}(x, t), A u_{2}\right\rangle \\
& +\frac{\sigma}{8}\left\|u_{2}\right\|_{2}^{2}+\frac{2}{\sigma}\left|f_{m}^{\prime}(x, t)\right|^{2},
\end{aligned}
$$

where $f_{m}(x, t)=\left(I-P_{m}\right) f(x, t)$.

Combining (48)-(51), we obtain from (47)

$$
\begin{aligned}
\frac{d}{d t}\left(\left\|v_{2}\right\|_{1}^{2}+\left\|u_{2}\right\|_{2}^{2}+2 k\left\langle\left(u^{+}\right)_{2}, A u_{2}\right\rangle\right. & +2\left\langle(g(u))_{2}, A u_{2}\right\rangle \\
\left.-2\left\langle f_{m}(x, t), A u_{2}\right\rangle\right)+\sigma( & \left\|v_{2}\right\|_{1}^{2}+\left\|u_{2}\right\|_{2}^{2} \\
& +2 k\left\langle\left(u^{+}\right)_{2}, A u_{2}\right\rangle \\
& +2\left\langle(g(u))_{2}, A u_{2}\right\rangle \\
& \left.-2\left\langle f_{m}(x, t), A u_{2}\right\rangle\right) \\
\leqslant \frac{8 k^{2}}{\sigma}\left|\left(u^{+}\right)_{2 t}\right|^{2}+\frac{8}{\sigma}\left|\left(g^{\prime}(u) u_{t}\right)_{2}\right|^{2} & +\frac{4}{\sigma}\left|f_{m}^{\prime}(x, t)\right|^{2}
\end{aligned}
$$

Like for (26)-(29), using the Hölder and Young inequalities, we get

$$
\begin{aligned}
& \frac{d}{d t}\left[\left|A u_{2}+k\left(u^{+}\right)_{2}+(g(u))_{2}-f_{m}(t)\right|^{2}+\left\|v_{2}\right\|_{1}^{2}\right] \\
& \quad+\sigma\left[\left|A u_{2}+k\left(u^{+}\right)_{2}+(g(u))_{2}-f_{m}(t)\right|^{2}+\left\|v_{2}\right\|_{1}^{2}\right] \\
& \leqslant \frac{8 k^{2}}{\sigma}\left|\left(u^{+}\right)_{2 t}\right|^{2}+\frac{8}{\sigma}\left|\left(g^{\prime}(u) u_{t}\right)_{2}\right|^{2}+\frac{4}{\sigma}\left|f_{m}^{\prime}(t)\right|^{2} \\
& \quad+2 k^{2}\left|\left(u^{+}\right)_{2}\right| \cdot\left|\left(u^{+}\right)_{2 t}\right|+2\left|(g(u))_{2}\right| \cdot\left|\left(g^{\prime}(u) u_{t}\right)_{2}\right| \\
& \quad+2\left|f_{m}(t)\right| \cdot\left|f_{m}^{\prime}(t)\right|+2\left|\left(g^{\prime}(u) u_{t}\right)_{2}\right| \cdot\left|f_{m}(t)\right| \\
& \quad+2\left|(g(u))_{2}\right| \cdot\left|f_{m}^{\prime}(t)\right|+2 k\left|\left(u^{+}\right)_{2 t}\right| \cdot\left|(g(u))_{2}\right|
\end{aligned}
$$

$$
\begin{aligned}
& +2 k\left|\left(u^{+}\right)_{2}\right| \cdot\left|\left(g^{\prime}(u) u_{t}\right)_{2}\right|+2 k\left|\left(u^{+}\right)_{2 t}\right| \cdot\left|f_{m}(t)\right| \\
& +2 k\left|\left(u^{+}\right)_{2}\right| \cdot\left|f_{m}^{\prime}(t)\right|+\sigma k^{2}\left|\left(u^{+}\right)_{2}\right|^{2}+\sigma\left|(g(u))_{2}\right|^{2} \\
& +\sigma\left|f_{m}(t)\right|^{2}+2 \sigma\left|(g(u))_{2}\right| \cdot\left|f_{m}(t)\right| \\
& +2 k \sigma\left|\left(u^{+}\right)_{2}\right| \cdot\left|(g(u))_{2}\right|+2 k \sigma\left|\left(u^{+}\right)_{2}\right| \cdot\left|f_{m}(t)\right| \\
\leqslant & 5 \sigma k^{2}\left|\left(u^{+}\right)_{2}\right|^{2}+\frac{11 k^{2}}{\sigma}\left|\left(u^{+}\right)_{2 t}\right|^{2}+5 \sigma\left|(g(u))_{2}\right|^{2} \\
& +\frac{11}{\sigma}\left|\left(g^{\prime}(u) u_{t}\right)_{2}\right|^{2}+\frac{9}{\sigma}\left|f_{m}(t)\right|^{2}+\frac{3}{\sigma}\left|f_{m}^{\prime}(t)\right|^{2} \\
\leqslant & l_{0}\left(\left|\left(u^{+}\right)_{2}\right|^{2}+\left|\left(u^{+}\right)_{2 t}\right|^{2}\right) \\
& +l_{1}\left(\left|(g(u))_{2}\right|^{2}+\left|\left(g^{\prime}(u) u_{t}\right)_{2}\right|^{2}\right) \\
& +l_{2}\left(\left|f_{m}(t)\right|^{2}+\left|f_{m}^{\prime}(t)\right|^{2}\right)
\end{aligned}
$$

where $l_{0}=\max \left\{5 \sigma k^{2}, 11 k^{2} / \sigma\right\}, l_{1}=\max \{5 \sigma, 11 / \sigma\}$, and $l_{2}=$ $\max \{9 / \sigma, 3 / \sigma\}$.

By the Gronwall lemma, we have

$$
\begin{aligned}
&\left|A u_{2}(t)\right|^{2}+\left|\Delta u_{2 t}(t)\right|^{2} \\
& \leqslant 4 l_{3} e^{-\sigma \tau}\left(\left|A u_{12}\right|^{2}+\left|\Delta u_{22}\right|^{2}\right) \\
&+8 l_{3} e^{-\sigma \tau}\left(2\left|(g(u))_{12}\right|^{2}+2\left|f_{m}(t-\tau)\right|^{2}\right) \\
&+4 l_{3}\left(k^{2}\left|\left(u^{+}\right)_{2}\right|^{2}+2\left|(g(u))_{2}\right|^{2}+2\left|f_{m}(t)\right|^{2}\right) \\
&+2 l_{0} l_{3} \int_{t-\tau}^{t} e^{-\sigma(t-s)}\left(\left|\left(u^{+}\right)_{2}\right|^{2}+\left|\left(u^{+}\right)_{2 t}\right|^{2}\right) d s \\
&+2 l_{1} l_{3} \int_{t-\tau}^{t} e^{-\sigma(t-s)}\left(\left|(g(u))_{2}\right|^{2}+\left|\left(g^{\prime}(u) u_{t}\right)_{2}\right|^{2}\right) d s \\
&+2 l_{2} l_{3} \int_{-\infty}^{t} e^{-\sigma(t-s)}\left(\left|f_{m}(s)\right|^{2}+\left|f_{m}^{\prime}(t)\right|^{2}\right) d s \\
&:= I_{1}+I_{2}+I_{3}+I_{4}+I_{5}+I_{6},
\end{aligned}
$$

where $l_{3}=\max \left\{1+2 \sigma^{2} \lambda^{-1}, 2\right\}$.

Then, given any $\widehat{D} \in \mathscr{D}_{\delta, \mathscr{E}_{1}}$, we have

$$
\left\|\phi_{2}\left(\tau, t-\tau, y_{0}\right)\right\|_{\mathscr{E}_{1}}^{2} \leqslant I_{1}+I_{2}+I_{3}+I_{4}+I_{5}+I_{6},
$$

for any $y_{0} \in D(t-\tau)$ and $\tau>0$.

Now we estimate $I_{1}, I_{2}, I_{3}, I_{4}, I_{5}$, and $I_{6}$ one by one. Given any $\epsilon>0$ and any $t \in \mathbb{R}$, first, by the definition of $\mathscr{D}_{\delta, \mathscr{E}_{1}}$, it is easy to see that there exists $\tau_{1} \geqslant 0$ such that, for $\tau \geqslant \tau_{1}$, $I_{1}, I_{2} \leqslant \epsilon / 6$.

Second, it is easy to see that

$$
\left|f_{m}(t)\right|^{2}=\left|\left(I-P_{m}\right) f(x, t)\right|^{2} \longrightarrow 0 \quad \text { as } n \longrightarrow \infty .
$$


By Theorem 8 , there exists $\tau_{2} \geqslant 0$ such that $\tau \geqslant \tau_{2},|\Delta u(t)|^{2} \leqslant$ $\left(R_{\delta, \mathscr{C}_{0}}(t)\right)^{2} \leqslant \infty$. Lemma 10 , we can choose $n_{1}$ large enough such that $I_{3} \leqslant \epsilon / 6$, for $n \geqslant n_{1}, \tau \geqslant \tau_{2}$.

Third, by Lemmas 10 and 11, we know that there exist $\tau_{3} \geqslant$ 0 and $n_{2}$ such that $I_{4}, I_{5} \leqslant \epsilon / 6$, for $n \geqslant n_{2}, \tau \geqslant \tau_{3}$.

Finally, by Lemma 9, we can choose $n_{3}$ large enough so that

$$
2 l_{2} l_{3} \int_{-\infty}^{t} e^{-\sigma(t-s)}\left(\left|f_{m}(s)\right|^{2}+\left|f_{m}^{\prime}(t)\right|^{2}\right) d s \leqslant \frac{\epsilon}{6}
$$

for $n \geqslant n_{3}$.

By the above analysis and (55), we know that, for any $\epsilon>$ 0 , there exist $\tau_{0}=\max \left\{\tau_{1}, \tau_{2}, \tau_{3}\right\}$ and $n_{0}=\max \left\{n_{1}, n_{2}, n_{3}\right\}$; then

$$
\left\|\phi_{2}\left(\tau, t-\tau, y_{0}\right)\right\|_{\mathscr{E}_{1}}^{2} \leqslant \epsilon
$$

for any $\tau>\tau_{0}, n>n_{0}$ and any $y_{0} \in D(t-\tau)$,

which implies the pullback $D_{\delta, \mathscr{E}_{1}}$-Condition $(C)$.

We complete the proof.

\section{Conflict of Interests}

The authors declare that there is no conflict of interests regarding the publication of this paper.

\section{Acknowledgments}

This work was partly supported by the NSFC 11361053, the NSFC 11101334, and the NWNU-LKQN-11-5.

\section{References}

[1] A. C. Lazer and P. J. Mckenna, "Large-amplitude periodic oscillations in suspension bridges. Some new connections with nonlinear analysis," SIAM Review, vol. 32, no. 4, pp. 537-578, 1990.

[2] Y. K. An, On the suspension bridge equations and the relevant problems [Ph.D. thesis], 2001.

[3] Y. An and C. Zhong, "Periodic solutions of a nonlinear suspension bridge equation with damping and nonconstant load," Journal of Mathematical Analysis and Applications, vol. 279, no. 2, pp. 569-579, 2003.

[4] Q. H. Choi and T. Jung, "A nonlinear suspension bridge equation with nonconstant load," Nonlinear Analysis: Theory, Methods \& Applications, vol. 35, no. 6, pp. 649-668, 1999.

[5] L. D. Humphreys, "Numerical mountain pass solutions of a suspension bridge equation," Nonlinear Analysis: Theory, Methods \& Applications, vol. 28, no. 11, pp. 1811-1826, 1997.

[6] A. C. Lazer and P. J. McKenna, "Large scale oscillatory behavior in asymmetric systems," Annales de l'Institut Henri Poincaré C, vol. 4, pp. 243-274, 1987.

[7] P. J. McKenna and W. Walter, "Nonlinear oscillations in a suspension bridge," Archive for Rational Mechanics and Analysis, vol. 98 , no. 2, pp. 167-177, 1987.

[8] Q. Z. Ma and C. K. Zhong, "Existence of global attractors for the coupled system of suspension bridge equations," Journal of Mathematical Analysis and Applications, vol. 308, no. 1, pp. 365379, 2005.
[9] Q. Z. Ma and C. K. Zhong, "Existence of global attractors for the suspension bridge equations," Journal of Sichuan University, vol. 43, no. 2, pp. 271-276, 2006.

[10] C. K. Zhong, Q. Z. Ma, and C. Y. Sun, "Existence of strong solutions and global attractors for the suspension bridge equations," Nonlinear Analysis: Theory, Methods \& Applications, vol. 67, no. 2, pp. 442-454, 2007.

[11] T. Caraballo, G. Łukaszewicz, and J. Real, "Pullback attractors for asymptotically compact non-autonomous dynamical systems," Nonlinear Analysis: Theory, Methods \& Applications, vol. 64, no. 3, pp. 484-498, 2006.

[12] J. Y. Park and J. R. Kang, "Pullback $\mathscr{D}$-attractors for nonautonomous suspension bridge equations," Nonlinear Analysis: Theory, Methods \& Applications, vol. 71, no. 10, pp. 4618-4623, 2009.

[13] J. R. Kang, "Pullback attractors for the non-autonomous coupled suspension bridge equations," Applied Mathematics and Computation, vol. 219, no. 16, pp. 8747-8758, 2013.

[14] Y. H. Wang and C. K. Zhong, "Pullback $\mathscr{D}$-attractors for nonautonomous sine-Gordon equations," Nonlinear Analysis: Theory, Methods \& Applications, vol. 67, no. 7, pp. 2137-2148, 2007. 


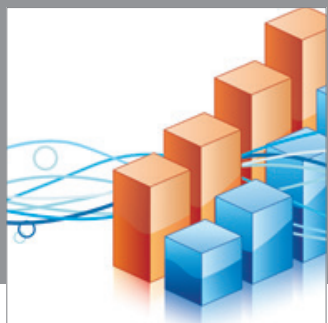

Advances in

Operations Research

mansans

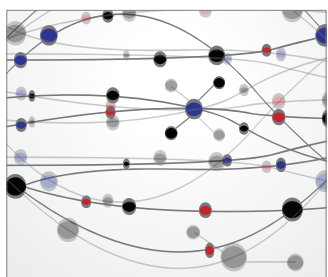

The Scientific World Journal
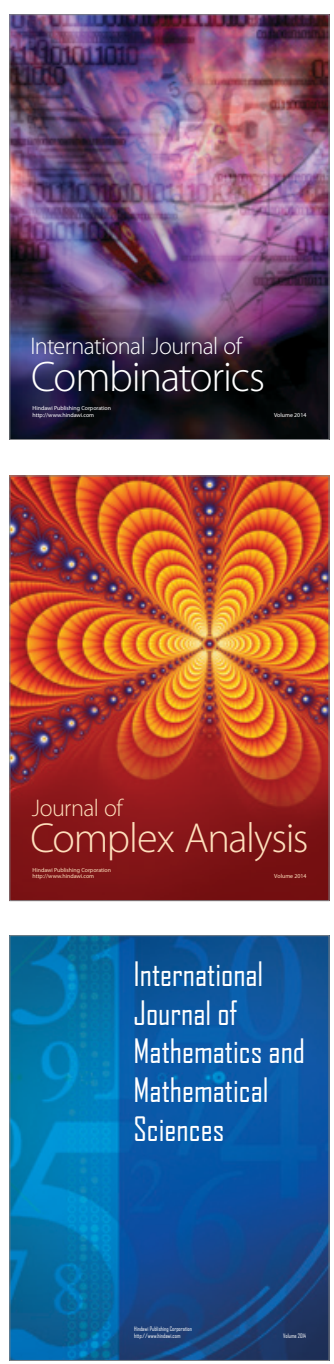
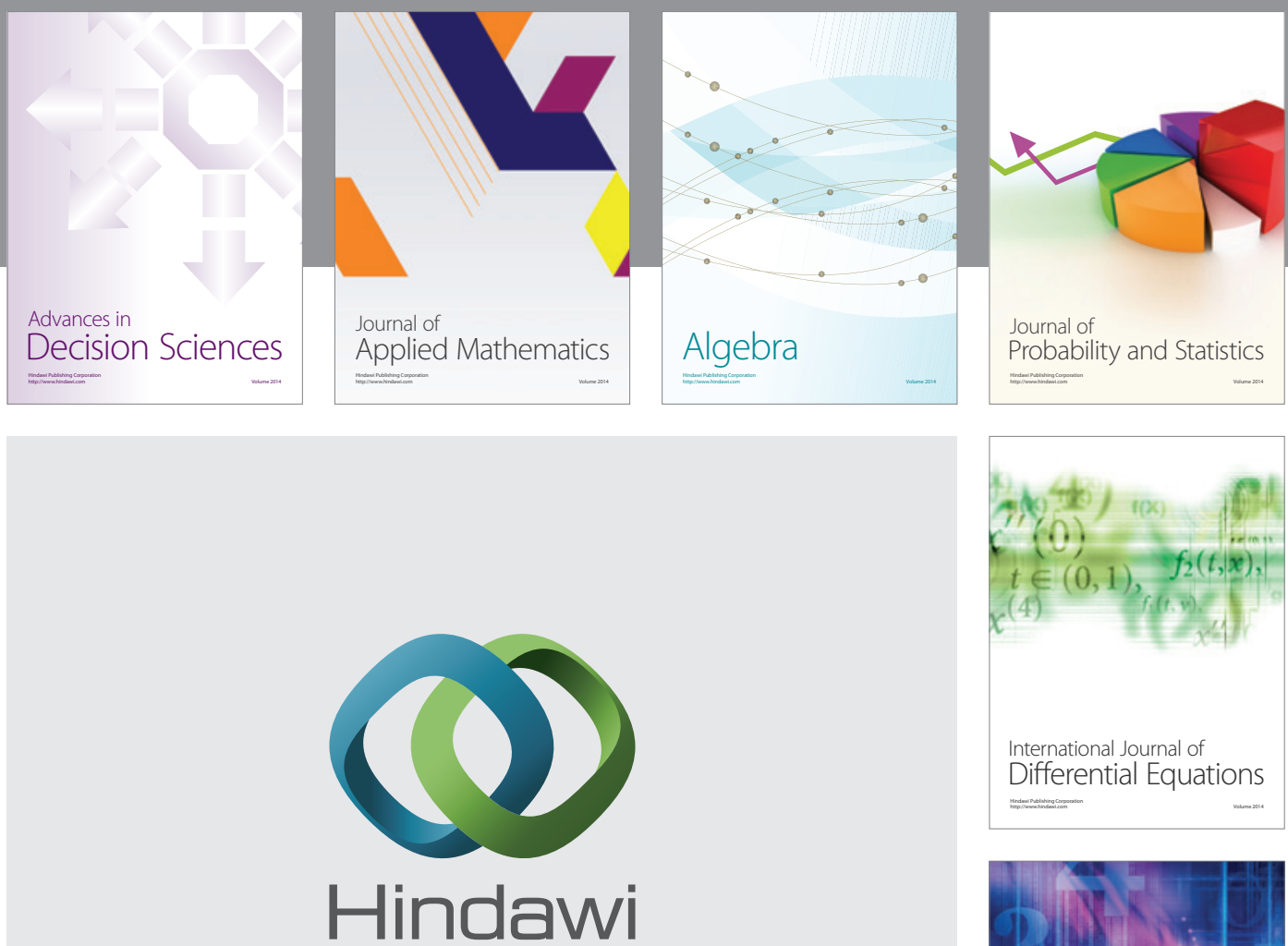

Submit your manuscripts at http://www.hindawi.com
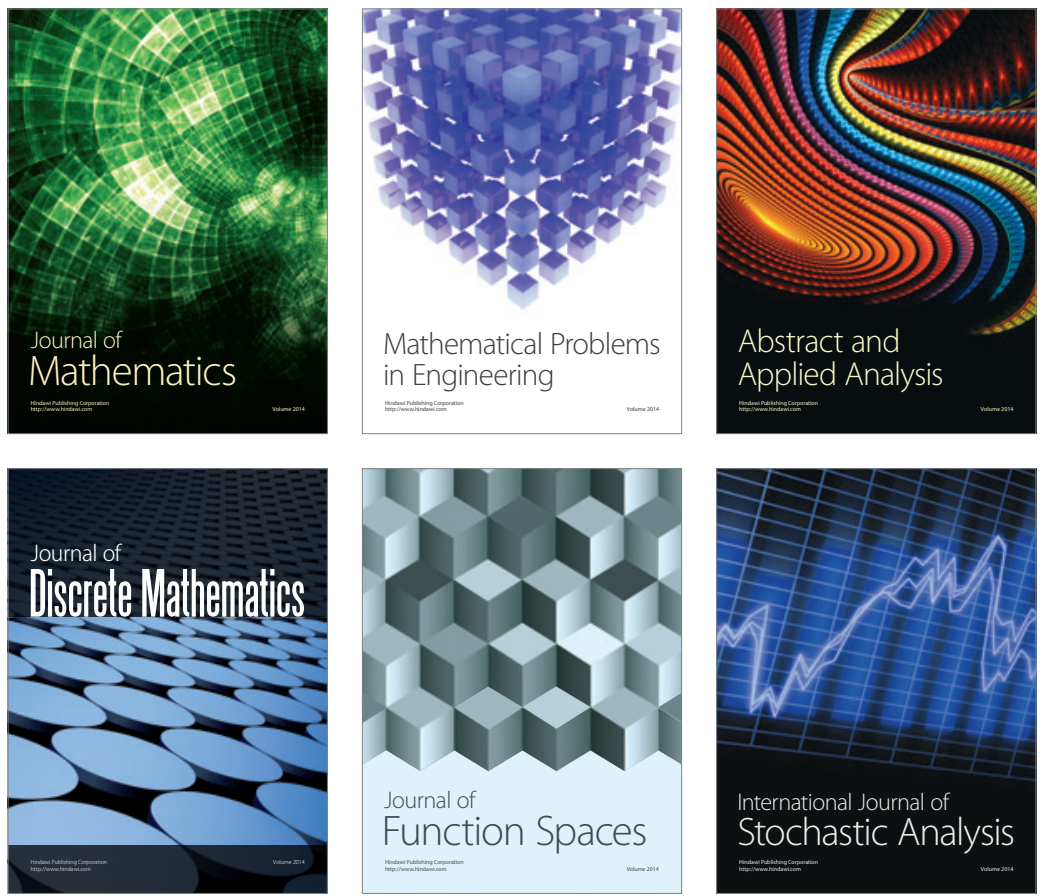

Journal of

Function Spaces

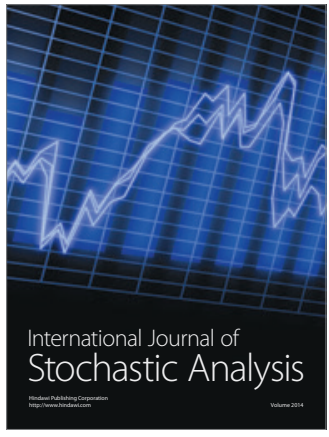

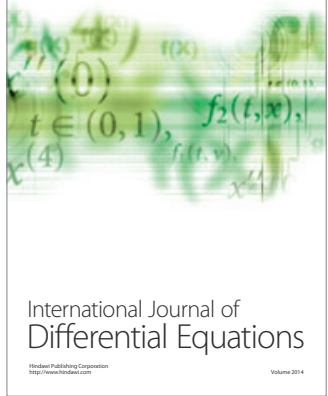
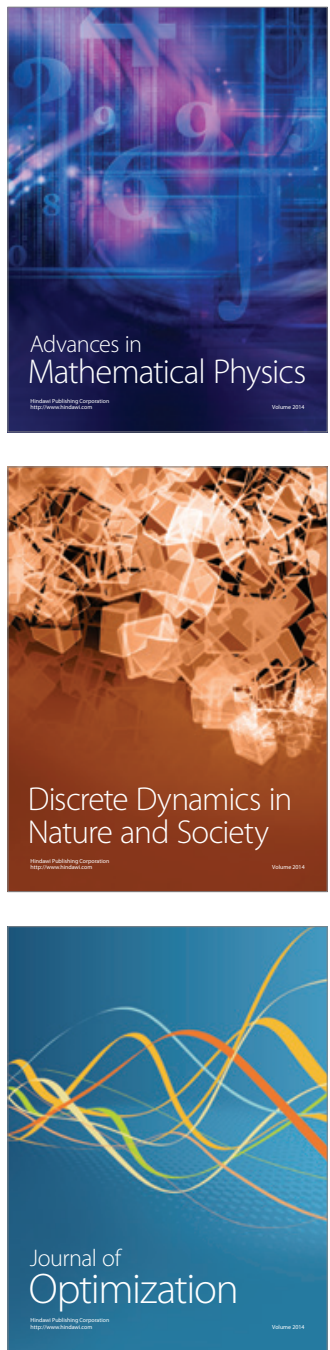